\title{
Real-World Study of Treatment with Pembrolizumab Among Patients with Advanced Urothelial Tract Cancer in Denmark
}

Lise Høj Omland ${ }^{\mathrm{a}}$, Dag Rune Stormoen ${ }^{\mathrm{a}, *}$, Line Hammer Dohn ${ }^{\mathrm{b}}$, Andreas Carus ${ }^{\mathrm{c}}$, Anne Birgitte Als ${ }^{\mathrm{d}}$, Niels Viggo Jensen ${ }^{\mathrm{e}}$, Gry Assam Taarnh $\varnothing \mathrm{j}^{\mathrm{a}}$, Anders Tolver ${ }^{\mathrm{f}}$ and Helle Pappot ${ }^{\mathrm{a}}$ ${ }^{a}$ Department of Oncology, Copenhagen University Hospital, Rigshospitalet, Copenhagen, Denmark

${ }^{\mathrm{b}}$ Department of Oncology, Herlev and Gentofte University Hospital, Copenhagen, Denmark

${ }^{\mathrm{c}}$ Department of Oncology, Aalborg University Hospital, Aalborg, Denmark

${ }^{\mathrm{d}}$ Department of Oncology, Aarhus University Hospital, Aarhus, Denmark

${ }^{\mathrm{e}}$ Department of Oncology, Odense University Hospital, Odense, Denmark

${ }^{\mathrm{f}}$ Department of Mathematical Sciences, Faculty of Science, University of Copenhagen, Copenhagen, Denmark

Received 28 March 2021

Accepted 25 June 2021

Pre-press 16 July 2021

Published 13 December 2021

\begin{abstract}
.
BACKGROUND: Investigating the effect of newly approved oncological drugs in the real-world is warranted. With emerging novel treatments rapidly being approved for urothelial tract cancers, we aimed to assess real-world data, regarding effect and safety, during the first year after approval of pembrolizumab in Denmark for patients with locally advanced and unresectable or metastatic urothelial tract cancer (mUTC) in the first- and second-line setting.

MATERIALS AND METHOD: At the six oncological departments treating mUTC in Denmark, we identified all mUTC patients receiving pembrolizumab during the first year after approval, between March 1, 2018 and February 28, 2019. A retrospective data collection was conducted from January to June 2020. Patient characteristics matching that of the relevant clinical trials for pembrolizumab in first- and second-line treatment-setting, overall survival (OS), progression-free survival (PFS), toxicity and tumor response were assessed.

RESULTS: 139 patients were identified, 53 in first-line treatment, 77 in second-line, and 9 receiving third or later lines of treatment. The population was characterized by a majority of males (70\%), most patients had ECOG PS 0-1 (60.4\%) and primary tumor in the bladder was predominant $(90.6 \%)$. The overall response rate (ORR) in first-line was $30.2 \%$, PFS was 3,5 months (95\% CI 2,3-7,9 months) and OS 9,2 months (95\% CI 7,0-20.9 months). For second-line treatment the ORR was $27,3 \%$, PFS 2,9 months (95\% CI 2,5-5,3) and OS 9.1 months (95\% CI 5,4-12,8 months). Toxicity was comparable to clinical trials without any new toxicities registered.
\end{abstract}

\footnotetext{
${ }^{*}$ Correspondence to: Dag Rune Stormoen, MD, Department of Oncology, Copenhagen University Hospital, Rigshospitalet, Blegdamsvej 9, DK-2100 Copenhagen, Denmark. Tel.: +45 35457969 ; E-mail: dag.rune.stormoen@ regionh.dk.
} 
CONCLUSION: Real-world data on response rates, OS, PFS and toxicity for patients with mUTC receiving pembrolizumab in first- and second-line, shows comparable results to clinical trials. This study further establishes immunotherapy as an effective and tolerable treatment for mUTC.

Keywords: Urothelial tract cancer, bladder cancer, immunotherapy, ICI, pembrolizumab, real-world treatment

\section{ABBREVIATIONS}

$\begin{array}{ll}\text { RW } & \text { Real-world } \\ \text { 1L } & \text { First-line } \\ \text { 2L } & \text { Second-line } \\ \text { IQR } & \text { Interquartile range } \\ \text { ECOG PS } & \begin{array}{l}\text { Eastern Cooperative Oncology } \\ \text { Group performance status }\end{array} \\ \text { PD-L1 } & \text { Programmed death-ligand 1 } \\ \text { ORR } & \text { Overall response rate } \\ \text { PFS } & \text { Progression-free survival } \\ \text { OS } & \text { Overall survival } \\ \text { mUTC } & \text { Metastatic urothelial tract cancer } \\ \text { CrCl } & \text { Creatinine Clearance }\end{array}$

\section{INTRODUCTION}

For decades, platinum-based chemotherapy has been the cornerstone of first-line treatment in patients with locally advanced, unresectable and metastatic urothelial tract cancer (mUTC) [1]. For fit patients with adequate renal function, standard treatment has been cisplatin-based combination chemotherapy, most often gemcitabine/cisplatin (GC) [1,2]. More than half of mUTC patients cannot, due to risk of high-grade toxicity, receive GC and are deemed cisplatin-ineligible [1, 2]. Recommended treatment for cisplatin-ineligible patients has been carboplatin-based combination chemotherapy, usually gemcitabine/carboplatin $(\mathrm{CaG})$ [3]. Response rates associated with $\mathrm{GC}$ and $\mathrm{CaG}$ are approximately $50 \%$ and $36 \%$, respectively $[2,4,5]$. However, the majority of patients will develop platinum-resistance and progressive disease within 12 months [6]. For years, numerous therapeutic agents have been evaluated in the second-line setting of mUTC treatment [7-10] and in Europe, vinflunine was for some years the only option approved for second-line treatment in platinum-resistant patients, while in the US taxanes were the recommended option $[11,12]$.

In Europe this changed in 2017 when the European Medicines Agency (EMA) approved the immunecheckpoint-inhibitors (ICIs) pembrolizumab, atezolizumab, and nivolumab as second-line treatment options in platinum-refractory advanced urothelial carcinoma patients based on phase-II and phase-III studies [6, 13-15]. Compared to existing secondline treatment options, pembrolizumab was superior in terms of overall survival (OS) (10.3 months vs. 7.4 months) and of overall response rate (ORR), complete response (CR) and partial response (PR) $[13,16]$. Subsequently, EMA also approved pembrolizumab and atezolizumab as first-line treatment options in cisplatin-ineligible mUTC patients based on two phase-II studies, [17, 18] but later restricted the approval to patients, whose cancers express programmed death-ligand 1 (PD-L1); defined as a combined positive score (CPS) of $\geq 10 \%$ in the case of pembrolizumab [11, 19]. The KEYNOTE-052 trial demonstrated an ORR of 24\% (CR 5\% and PR $19 \%$ ) and a disease control rate (DCR) of 47\% [17]. Long-term follow-up data demonstrated even more favorable outcomes with an ORR of $28.6 \%$ (CR 8.9\% and PR 19.7\%), a DCR of $46.8 \%$ and median OS of 11.3 months in the total population [20].

Pembrolizumab and atezolizumab have been approved as first-line treatment; pembrolizumab, atezolizumab and nivolumab as second-line treatment in mUTC patients in Denmark following EMA approval. However, pembrolizumab was the only available option for use in Danish hospitals in the research period due to local health economic decisions [21].

Although the results from the clinical trials evaluating pembrolizumab are promising, effectiveness and safety of pembrolizumab in mUTC patients treated in the real-world clinical setting is unknown. Due to strict eligibility criteria, patients enrolled in clinical trials are often younger and without comorbidities or generalized organ dysfunction [22]. The disadvantage of such strict eligibility criteria is that the results from clinical trials are not generalizable to all populations [23, 24]. Real-world evidence is healthcare data derived from multiple sources outside the context of traditional clinical studies including data obtained from electronic medical records [24]. Realworld evidence provides information on treatment effectiveness and safety in the general population and thus complement the evidence emerged from clinical studies [23]. This may be particularly important in 
mUTC patients, representing a heterogenous group in terms of performance status and comorbidities [25]. mUTC comprises kidney pelvic, ureters, bladder and urethral cancers.

We therefore performed a nationwide, populationbased cohort study to evaluate effectiveness, safety and survival in mUTC patients treated with pembrolizumab in the real-world clinical setting at Danish oncology departments the first year after implementation of pembrolizumab as standard treatment in Denmark. Further we aimed to compare our findings with registration studies for pembrolizumab in the first- and second-line setting [16, 20].

\section{MATERIAL AND METHODS}

\section{Treatment setting}

Each year, nearly 1100 patients are diagnosed with $\geq \mathrm{T} 1$ urothelial tract cancer in Denmark [26]. Approximately $30 \%$ of these patients eventually develop muscle-invasive disease, the number of patients progressing to disseminated disease is unknown [27].

Danish residents are equally entitled to publicly funded health care services. Six specialized urooncology departments manage treatment of mUTC patients in Denmark [26]. Privately financed health care is limited and does not include oncological treatment of mUTC [28-30].

According to Danish mUTC treatment guidelines, cisplatin-ineligible patients are defined as patients with a creatinine clearance $(\mathrm{CrCl})<50 \mathrm{ml} / \mathrm{min}$, an Eastern Cooperative Oncology Group (ECOG) performance status (PS) $>1$, a New York Heart Association (NYHA) class $>2$ heart failure, significant hearing loss and/or neuropathy. First-line pembrolizumab can be offered to cisplatin-ineligible patients provided that they have $\mathrm{CrCl}$ of $>30 \mathrm{ml} / \mathrm{min}$, an ECOG PS of 0-2, a PD-L1 combined positive score (CPS) of $\geq 10$, no evidence of active chronic viral infection, no autoimmune disease (except well controlled diabetes, thyroid disease or arthritis) and no uncontrolled intracerebral metastases. Further, they may not receive more than the equivalent of 10 $\mathrm{mg}$ prednisone daily. Pembrolizumab is the recommended second-line treatment following progression during or after platinum-based chemotherapy regardless of PD-L1 expression [29]. According to national treatment guidelines surveillance scans are performed with computed tomography (CT) and planned after every 3rd treatment series.

\section{Study population and data collection}

We identified mUTC patients, from the six relevant oncologic departments in Denmark, that initiated pembrolizumab between March 1, 2018 and February 28, 2019 and had no prior ICI-treatment for mUTC. Data were obtained from electronic medical records by uro-oncologists at each department and included in a REDCap database. Data were collected from January to June 2020.

Patients were categorized according to renal function, ECOG PS, PD-L1 expression, previous neoadjuvant chemotherapy, and whether progressing to mUTC less than 12 months after end of neoadjuvant chemotherapy. PD-L1 analysis results were from most recent biopsy, either primary tumor or metastasis, when performed.

The study was approved by the Danish Patient Safety Authorities (file no.: 3-3013/3010/1) and the Danish Data Protection Agency (file no.: VD-2019175 ) and was conducted according to the Declaration of Helsinki. For retrospective data retrieval as described above, ethics committee approval and patient consent is not required in Denmark [31].

\section{Outcomes}

\section{Definition of tumor responses}

Evaluation of tumor response was performed by local radiologists at each center with planned CT scans, in accordance with local clinical guidelines. ORR was defined as the proportion of patients achieving $\mathrm{CR}$ or PR. DCR was defined as the proportion of patients with CR, PR or stable disease (SD). Time to response was defined as the time from first pembrolizumab treatment to first radiological documentation of CR or PR. Duration of response was the time from first radiologically demonstrated CR or $\mathrm{PR}$ to radiological progression or death. Radiological response and progression were defined by the radiologist, according to local practice at each treatment center.

\section{Definition of PFS and OS}

Progression-free survival (PFS) was defined as the time from first pembrolizumab treatment to the date of radiological progression or death from any cause. Patients who did not have a radiological evaluation performed were excluded from PFS analysis. OS was defined as the period from first pembrolizumab treatment to the date of death from any cause. 


\section{Toxicity}

Toxicity was graded according to the National Cancer Institute's (NCI) Common Terminology Criteria for Adverse Events (CTCAE). Grade 3 and 4 toxicities were registered.

\section{Statistical analysis}

Time of follow-up was calculated as the time from first pembrolizumab treatment to the date of last data entry. Patients without CT scans were counted as "no radiographic evaluation performed". Median and range of time to response was reported for patients demonstrating CR or PR. Duration of response, progression free survival (PFS) and overall survival (OS) were treated as right censored survival data. The Kaplan-Meier method was used for estimation of survival, $95 \%$ confidence intervals (CI) for median survival and survival probability at 3, 6 and 12 months. Due to censoring, estimation of a CI upper limit for median duration of response was not possible (indicated by NA). Statistical analyses were carried out using R in particular the "survival" and the "prodlim" packages

\section{RESULTS}

A total of 139 patients were included. Fiftythree patients received pembrolizumab as first-line treatment and 77 patients received pembrolizumab as second-line treatment; nine patients received pembrolizumab in the third-line setting. Patient characteristics are presented in Table 1.

Briefly, median age at initiation of pembrolizumab treatment was 71 years (IQR: 68-78 years) in the firstline setting, and 69 years (IQR: 65-75 years) in the second-line setting. Most patients were male (70.5\%) and the majority had the primary tumor located in the bladder $(90,6 \%)$ with urothelial carcinoma as the predominant histologic subtype. For patients treated with first-line pembrolizumab, more than half had ECOG PS 0-1 (60.4\%) and a third had ECOG PS $2(34 \%)$, In the second-line and third-line settings, $81.8 \%$ and $88.9 \%$, respectively, had an ECOG PS of $0-1$.

Seventeen of 53 patients $(32.1 \%)$ treated with first-line pembrolizumab, previously received neoadjuvant chemotherapy. Of these 17 patients, 13 progressed (76\%) to mUTC within 12 months after end of neoadjuvant chemotherapy.

Renal function was assessed in most patients prior to pembrolizumab initiation (98.6\%). For most patients receiving first- and second-line treatment, $\mathrm{CrCl}$ was $<60 \mathrm{ml} / \mathrm{min}$.

In the total cohort, the PD-L1 testing rate was $13.7 \%$; in patients treated with first-line pembrolizumab the testing rate was $26.4 \%$ compared to $6.5 \%$ and $0.0 \%$ in patients receiving pembrolizumab as second-line and beyond second-line treatment, respectively. Of patients tested, most had a PD-L1 expression $\geq 10 \%(89,5 \%)$.

\section{Tumor responses}

Patients treated with first-line pembrolizumab received a median of four treatment cycles (Table 2).

ORR was $30.2 \%$ and time to response was 1.9 months (range 1.7-8.0 months). Median duration of response was 16.9 months (95\% CI: 5.9 months - not reached) and the probability of responses lasting at least 12 months was $62.5 \%$ [95\% CI: $38.8-76.2 \%$ ). Disease control was achieved in 23 of 53 patients $(43.4 \%)$. In patients receiving second-line pembrolizumab, a median of four treatment cycles were administered. 21 of 77 (27.3\%) patients had an objective response and the DCR was $42.9 \%$. Time to response was 2.0 months (range 1.2-3.9 months). Median duration of response was 12.9 months (95\% CI: 5.1 months - not reached) and $57.1 \%$ (95\% CI: $36.0-78.3 \%)$ of responses lasted a minimum of 12 months.

At the time of data cutoff, nine of 139 patients $(6.5 \%)$ were still receiving pembrolizumab. In all three treatment settings, the main reason for pembrolizumab discontinuation was progressive disease.

\section{PFS and $O S$}

Median PFS in patients treated with first-line pembrolizumab ( $n=53$ ) was 3.5 months (95\% CI: $2.3-7.9$ months). Eleven patients died prior to first evaluation scan. The estimated PFS rate at 3, 6 and 12 months was $50.9 \%$ (95\% CI: $37.5-64.4$ ), $39.6 \%$ (95\% CI: 26.5-52.8) and 22.6 (95\% CI: 11.4-33.9), respectively. In the second-line setting, 13 patients died within three months after treatment initiation and seven of these were not evaluated radiologically. Median PFS was 2.9 months (95\% CI: 2.5-5.3 months) and the 3, 6 and 12 months PFS rate was $49.4 \%$ (95\% CI: 38.2-60.5), 32.5\% (95\% CI: 22.0-42.9) and 20.8\% (95\% CI: 11.7-29.8). OS and PFS data are shown in Table 3.

For patients receiving first-line pembrolizumab $(n=53)$, median OS was 9.2 months (95\% CI: 
Table 1

Baseline characteristics of 139 mUTC patients initiating pembrolizumab at Danish oncology departments from March 1, 2018 to February 28, 2019

\begin{tabular}{|c|c|c|c|c|}
\hline & All $(n=139)$ & $1 \mathrm{~L}(n=53)$ & $2 \mathrm{~L}(n=77)$ & $>2 \mathrm{~L}(n=9)$ \\
\hline $\begin{array}{l}\text { Age at diagnosis of mUTC, } \\
\text { years, median (IQR) }\end{array}$ & $68(64-75)$ & $70(67-77)$ & $67(63-74)$ & $66(63-71)$ \\
\hline $\begin{array}{l}\text { Age at pembrolizumab initiation, } \\
\text { years, median (IQR) }\end{array}$ & $71(66-76)$ & $71(68-78)$ & $69(65-75)$ & $72(65-73)$ \\
\hline \multicolumn{5}{|l|}{ Age categories at pembrolizumab initiation } \\
\hline$<50$ years, no $(\%)$ & $1(0.7)$ & $0(0.0)$ & $1(1.3)$ & $0(0.0)$ \\
\hline $50-64$ years, no $(\%)$ & $25(18.0)$ & $8(15.1)$ & $15(19.5)$ & $2(22.2)$ \\
\hline $65-74$ years, no (\%) & $72(51.8)$ & $27(50.9)$ & $40(51.9)$ & $5(55.6)$ \\
\hline$\geq 75$ years, no $(\%)$ & $41(29.5)$ & $18(34.0)$ & $21(27.3)$ & $2(22.2)$ \\
\hline \multicolumn{5}{|l|}{ Gender } \\
\hline Male, no (\%) & $98(70.5)$ & $36(67.9)$ & $56(72.7)$ & $6(66.7)$ \\
\hline Female, no (\%) & $41(29.5)$ & $17(32.1)$ & $21(27.3)$ & $3(33.3)$ \\
\hline \multicolumn{5}{|l|}{ Smoking status } \\
\hline History of smoking, no (\%) & $101(72.7)$ & $37(69.8)$ & $58(75.3)$ & $6(66.7)$ \\
\hline No history of smoking, no (\%) & $21(15.1)$ & $9(17.0)$ & $9(11.7)$ & $3(33.3)$ \\
\hline Unknown, no (\%) & $17(12.2)$ & $7(13.2)$ & $10(13.0)$ & $0(0.0)$ \\
\hline \multicolumn{5}{|l|}{ Location of primary tumor } \\
\hline Upper urinary tract, no (\%) & $31(22.3)$ & $5(9.4)$ & $26(33.8)$ & $0(0.0)$ \\
\hline Bladder, no $(\%)$ & $106(76.3)$ & $48(90.6)$ & $51(66.2)$ & $7(77.8)$ \\
\hline Urethra, no $(\%)$ & $1(0.7)$ & $0(0.0)$ & $0(0.0)$ & $1(11.1)$ \\
\hline Unknown, no (\%) & $1(0.7)$ & $0(0.0)$ & $0(0.0)$ & $1(11.1)$ \\
\hline \multicolumn{5}{|l|}{ Histology } \\
\hline Urothelial carcinoma, no (\%) & $132(95.0)$ & $50(94.3)$ & $73(94.8)$ & $9(100)$ \\
\hline Squamous cell carcinoma, no (\%) & $3(2.2)$ & $1(1.9)$ & $2(2.6)$ & $0(0.0)$ \\
\hline Adenocarcinoma, no (\%) & $0(0.0)$ & $0(0.0)$ & $0(0.0)$ & $0(0.0)$ \\
\hline Mixed histology, no (\%) & $3(2.2)$ & $2(3.8)$ & $1(1.3)$ & $0(0.0)$ \\
\hline Other, no $(\%)$ & $1(0.7)$ & $0(0.0)$ & $1(1.3)$ & $0(0.0)$ \\
\hline \multicolumn{5}{|l|}{ ECOG PS at pembrolizumab initiation } \\
\hline 0, no $(\%)$ & $35(25.2)$ & $14(26.4)$ & $18(23.4)$ & $3(33.3)$ \\
\hline 1, no $(\%)$ & $68(48.9)$ & $18(34.0)$ & $45(58.4)$ & $5(55.6)$ \\
\hline 2, no $(\%)$ & $25(18.0)$ & $18(34.0)$ & $7(9.1)$ & $0(0.0)$ \\
\hline 3, no $(\%)$ & $0(0.0)$ & $0(0.0)$ & $0(0.0)$ & $0(0.0)$ \\
\hline Unknown, no (\%) & $11(7.9)$ & $3(5.7)$ & $7(9.1)$ & $1(11.1)$ \\
\hline \multicolumn{5}{|l|}{ Prior treatments } \\
\hline Neoadjuvant chemotherapy, no (\%) & $23(16.5)$ & $17(32.1)$ & $6(7.8)$ & $0(0.0)$ \\
\hline Cystectomy, no (\%) & $50(36.0)$ & $22(41.5)$ & $25(32.5)$ & $3(33.3)$ \\
\hline Nephroureterectomy, no (\%) & $17(12.2)$ & $2(3.8)$ & $15(19.5)$ & $0(0.0)$ \\
\hline 1L chemotherapy, no $(\%)$ & $86(61.9)$ & $0(0.0)$ & $77(100)$ & $9(100)$ \\
\hline 2L chemotherapy, no $(\%)$ & $9(6.5)$ & $0(0.0)$ & $0(0.0)$ & $9(100)$ \\
\hline Radiotherapy to bladder, no (\%) & $13(9.4)$ & $7(13.2)$ & $5(6.5)$ & $1(11.1)$ \\
\hline No prior treatment, no $(\%)$ & $21(15.1)$ & $21(39.6)$ & $0(0.0)$ & $0(0.0)$ \\
\hline \multicolumn{5}{|l|}{ Metastatic sites at pembrolizumab initiation } \\
\hline Lymph nodes below the diaphragm, no (\%) & $75(54.0)$ & $28(52.8)$ & $40(51.9)$ & $7(77.8)$ \\
\hline Lymph nodes above the diaphragm, no (\%) & $37(26.6)$ & $12(22.6)$ & $23(29.9)$ & $2(22.2)$ \\
\hline Liver, no $(\%)$ & $28(20.1)$ & $7(13.2)$ & $20(26.0)$ & $1(11.1)$ \\
\hline Lungs, no $(\%)$ & $51(36.7)$ & $15(28.3)$ & $35(45.5)$ & $1(11.1)$ \\
\hline Bone, no $(\%)$ & $42(30.2)$ & $17(32.1)$ & $25(32.5)$ & $0(0.0)$ \\
\hline Brain, no $(\%)$ & $1(0.7)$ & $0(0.0)$ & $1(1.3)$ & $0(0.0)$ \\
\hline Adrenal gland, no (\%) & $9(6.5)$ & $5(9.4)$ & $4(5.2)$ & $0(0.0)$ \\
\hline Pelvis, no $(\%)$ & $18(12.9)$ & $7(13.2)$ & $11(14.3)$ & $0(0.0)$ \\
\hline Carcinosis, no $(\%)$ & $19(13.7)$ & $6(11.3)$ & $10(13.0)$ & $3(33.3)$ \\
\hline Other locations, no $(\%)$ & $22(15.8)$ & $9(17.0)$ & $12(15.6)$ & $1(11.1)$ \\
\hline No metastases, no (\%) & $1(0.7)$ & $1(1.9)$ & $0(0.0)$ & $0(0.0)$ \\
\hline $\begin{array}{l}\text { Hemoglobin tested prior to } \\
\text { pembrolizumab initiation, no }(\%)\end{array}$ & 137 (98.6) & $52(98.1)$ & $76(98.7)$ & $9(100)$ \\
\hline $\begin{array}{l}\text { Hemoglobin level } \\
\quad \text { (in patients tested), median (range) }\end{array}$ & $7.2(4.8-10.0)$ & $6.9(4.8-10.0)$ & $7.3(5.3-9.4)$ & $7.8(6.2-9.1)$ \\
\hline $\begin{array}{l}\text { GFR tested prior to pembrolizumab } \\
\text { initiation, no }(\%)\end{array}$ & 137 (98.6) & $52(98.1)$ & $76(98.7)$ & $9(100)$ \\
\hline
\end{tabular}


Table 1

(Continued)

\begin{tabular}{|c|c|c|c|c|}
\hline & All $(n=139)$ & $1 \mathrm{~L}(n=53)$ & $2 \mathrm{~L}(n=77)$ & $>2 \mathrm{~L}(n=9)$ \\
\hline \multicolumn{5}{|l|}{ Type of GFR test (in patients tested) } \\
\hline${ }^{51}$ Cr-EDTA, no (\%) & $39(28.5)$ & $33(63.5)$ & $6(7.9)$ & $0(0.0)$ \\
\hline eGFR, no (\%) & $98(71.5)$ & $19(36.5)$ & $70(92.1)$ & $9(100)$ \\
\hline \multicolumn{5}{|l|}{ GFR (in patients tested), no (\%) } \\
\hline$<15 \mathrm{ml} / \mathrm{min}$ & $1(0.7)$ & $1(1.9)$ & $0(0.0)$ & $0(0.0)$ \\
\hline $15-29 \mathrm{ml} / \mathrm{min}$ & $5(3.6)$ & $2(3.8)$ & $3(3.9)$ & $0(0.0)$ \\
\hline $30-49 \mathrm{ml} / \mathrm{min}$ & $41(29.9)$ & $19(36.5)$ & $20(26.3)$ & $2(22.2)$ \\
\hline $50-59 \mathrm{ml} / \mathrm{min}$ & $25(18.2)$ & $6(11.5)$ & $17(22.4)$ & $2(22.2)$ \\
\hline$\geq 60 \mathrm{ml} / \mathrm{min}$ & $65(47.4)$ & $24(46.2)$ & $36(47.4)$ & $5(55.6)$ \\
\hline $\begin{array}{l}\text { PD-L1 expression tested prior } \\
\text { to pembrolizumab initiation, no }(\%)\end{array}$ & $19(13.7)$ & $14(26.4)$ & $5(6.5)$ & $0(0.0)$ \\
\hline \multicolumn{5}{|l|}{$\begin{array}{l}\text { PD-L1 expression } \\
\quad \text { (in patients tested), no (\%) }\end{array}$} \\
\hline$<1 \%$ & $2(10.5)$ & $1(7.1)$ & $1(20.0)$ & $0(0.0)$ \\
\hline $1-9 \%$ & $0(0.0)$ & $0(0.0)$ & $0(0.0)$ & $0(0.0)$ \\
\hline$\geq 10 \%$ & $17(89.5)$ & $13(92.9)$ & $4(80.0)$ & $0(0.0)$ \\
\hline
\end{tabular}

Abbreviations: $1 \mathrm{~L}=$ first-line; $2 \mathrm{~L}=$ second-line; $>2 \mathrm{~L}=$ beyond second-line; $\mathrm{mUTC}=$ metastatic urothelial tract cancer; IQR $=$ interquartile range; ECOG PS = Eastern Cooperative Oncology Group performance status; GFR = glomerular filtration rate; ${ }^{51} \mathrm{Cr}-\mathrm{EDTA}=\mathrm{chromium}-51$ labeled ethylenediamine tetraacetic acid clearance; eGFR = estimated GFR; PD-L1 = Programmed death-ligand 1.

7.0-20.9 months) and survival at 3, 6 and 12 months was $79.2 \%$ (95\% CI: 68.3-90.2), 66.0\% (95\% CI: 53.3-78.8) and 45.3\% (95\% CI: 31.9-58.7). Among patients treated with pembrolizumab in the secondline setting $(n=77)$, median OS was 9.1 months $(95 \%$ CI: 5.4-12.8 months); survival at 3, 6 and 12 months was $83.1 \%$ (95\% CI: $74.7-91.5$ ), $59.7 \%$ (95\% CI: 48.8-70.7) and 40.0 (95\% CI: 29.0-51.0). OS and PFS are demonstrated in Fig. 1 and 2, respectively. In Fig. 3, we demonstrate OS divided by treatment line.

\section{Toxicity}

In the first-line setting, $20.8 \%$ had at least one treatment-related grade $3 \mathrm{AE}$ and $1.9 \%$ of patients grade $4 \mathrm{AE}$. The most common types of AE grade 3 were colitis $(5.7 \%)$ and pneumonitis $(5.7 \%)$. The only registered grade $4 \mathrm{AE}$ in first-line setting was thrombotic microangiopathy $(n=1)$. Among patients treated with second-line pembrolizumab, $16.9 \%$ experienced at least one treatment-related $\mathrm{AE}$ grade 3 ; most commonly colitis $(2.6 \%)$, pneumonitis (2.6\%), adrenal insufficiency $(2.6 \%)$ and pneumonia (2.6\%). One case of pneumonitis grade 4 was registered in patients treated in the second-line setting. No treatment-related deaths were registered. In the total patient cohort, approximately $20 \%$ of patients discontinued pembrolizumab due to toxicity. Registered toxicities are shown in Table 2.

\section{DISCUSSION}

In this population-based, nationwide retrospective study, we examined real-world data, including
PFS, OS and safety, following implementation of pembrolizumab as standard treatment for mUTC in Denmark. This is, to the best of our knowledge, the first study to evaluate treatment patterns and survival for mUTC patients treated with pembrolizumab in the real-world setting and compare this with relevant clinical trials.

Patient characteristics were comparable in our cohort and in the clinical trials, with exception of age and liver metastasis at inclusion. The main findings from KEYNOTE-045, KEYNOTE-052 and the present study are presented in Table 4 for comparison.

In our patient cohort, median age of patients initiating first-line pembrolizumab was three years lower than the median age of patients included in the clinical trial KEYNOTE-052, 71 and 74 years, respectively. More patients enrolled in KEYNOTE-052 had liver metastases at baseline ( $21 \%$ vs $13.2 \%)$. This is surprising, as patients enrolled in clinical trials are often younger and more fit than patients treated in the realworld clinical setting. [22] A possible explanation for this could be that some oncologists in real-life have refrained from treating elderly patients with ICI, due to a limited amount of knowledge of treatment efficacy and side effects in the elderly patient group. Patients in the present study receiving second-line pembrolizumab were slightly older and more patients had ECOG PS 2 compared to patients enrolled in the clinical trial KEYNOTE-045, but more patients in the clinical trial had liver metastases. This is of note, as patients with ECOG PS 2 and liver metastases were excluded from enrolment in KEYNOTE-045. In our cohort of patients treated in the first-line setting, there 
Table 2

Tumor responses and toxicity to pembrolizumab in 139 mUTC patients initiating pembrolizumab at Danish oncology departments between March 1, 2018 and February 28, 2019

\begin{tabular}{|c|c|c|c|c|}
\hline & All $(n=139)$ & $1 \mathrm{~L}(n=53)$ & $2 \mathrm{~L}(n=77)$ & $>2 \mathrm{~L}(n=9)$ \\
\hline $\begin{array}{l}\text { Number of cycles of } \\
\text { pembrolizumab, median (range) }\end{array}$ & $4(1-34)$ & $4(1-34)$ & $4(1-32)$ & $9(2-30)$ \\
\hline \multicolumn{5}{|l|}{ Best overall response } \\
\hline Complete response, no (\%) & $6(4.3)$ & $2(3.8)$ & $4(5.2)$ & $0(0.0)$ \\
\hline Partial response, no $(\%)$ & $35(25.2)$ & $14(26.4)$ & $17(22.1)$ & $4(44.4)$ \\
\hline Stable disease, no $(\%)$ & $21(15.1)$ & $7(13.2)$ & $12(15.6)$ & $2(22.2)$ \\
\hline Progressive disease, no $(\%)$ & $53(38.1)$ & $18(34.0)$ & $32(41.6)$ & $3(33.3)$ \\
\hline Mixed response, no (\%) & $3(2.2)$ & $0(0.0)$ & $3(3.9)$ & $0(0.0)$ \\
\hline No radiologic evaluation performed, no $(\%)$ & $18(12.9)$ & $11(20.8)$ & $7(9.1)$ & $0(0.0)$ \\
\hline Not evaluable, no $(\%)$ & $3(2.2)$ & $1(1.9)$ & $2(2.6)$ & $0(0.0)$ \\
\hline Time to response, months, median (range) & $1.9(1.2-8.0)$ & $1.9(1.7-8.0)$ & $2.0(1.2-3.9)$ & $1.6(1.5-5.1)$ \\
\hline $\begin{array}{l}\text { Duration of response, months, } \\
\text { median }(95 \% \mathrm{CI})\end{array}$ & $16.7(11.5-\mathrm{NA})$ & $16.9(5.9-\mathrm{NA})$ & $12.9(5.1-\mathrm{NA})$ & - \pm \\
\hline CTCAE 5.0 AE grade 3 , no $(\%)^{\infty}$ & $25(18.0)$ & $11(20.8)$ & $13(16.9)$ & $1(11.1)$ \\
\hline \multicolumn{5}{|l|}{ Type of AE grade $3 x$} \\
\hline Colitis, no $(\%)$ & $5(3.6)$ & $3(5.7)$ & $2(2.6)$ & $0(0.0)$ \\
\hline Pneumonitis, no $(\%)$ & $5(3.6)$ & $3(5.7)$ & $2(2.6)$ & $0(0.0)$ \\
\hline Adrenal insufficiency, no (\%) & $3(2.2)$ & $1(1.9)$ & $2(2.6)$ & $0(0.0)$ \\
\hline Hypophysitis, no (\%) & $3(2.2)$ & $2(3.8)$ & $1(1.3)$ & $0(0.0)$ \\
\hline Liver affection, no $(\%)$ & $2(1.4)$ & $1(1.9)$ & $1(1.3)$ & $0(0.0)$ \\
\hline Nephritis, no (\%) & $2(1.4)$ & $1(1.9)$ & $0(0.0)$ & $1(11.1)$ \\
\hline Diarrhea, no $(\%)$ & $2(1.4)$ & $1(1.9)$ & $1(1.3)$ & $0(0.0)$ \\
\hline Allergic reaction, no (\%) & $1(0.7)$ & $1(1.9)$ & $0(0.0)$ & $0(0.0)$ \\
\hline Constipation, no (\%) & $1(0.7)$ & $0(0.0)$ & $1(1.3)$ & $0(0.0)$ \\
\hline Itching, no $(\%)$ & $1(0.7)$ & $1(1.9)$ & $0(0.0)$ & $0(0.0)$ \\
\hline Skin rash, no (\%) & $1(0.7)$ & $1(1.9)$ & $0(0.0)$ & $0(0.0)$ \\
\hline Arthritis, no (\%) & $1(0.7)$ & $0(0.0)$ & $1(1.3)$ & $0(0.0)$ \\
\hline Pneumonia, no $(\%)$ & $2(1.4)$ & $0(0.0)$ & $2(2.6)$ & $0(0.0)$ \\
\hline Neuritis, no $(\%)$ & $1(0.7)$ & $0(0.0)$ & $1(1.3)$ & $0(0.0)$ \\
\hline Upper and lower extremity edema, no (\%) & $1(0.7)$ & $1(1.9)$ & $0(0.0)$ & $0(0.0)$ \\
\hline CTCAE 5.0 AE grade 4 , no $(\%)^{\infty}$ & $2(1.4)$ & $1(1.9)$ & $1(1.3)$ & $0(0.0)$ \\
\hline \multicolumn{5}{|l|}{ Type of AE grade $4 x$} \\
\hline Pneumonitis, no (\%) & $1(0.7)$ & $0(0.0)$ & $1(1.3)$ & $0(0.0)$ \\
\hline Thrombotic microangiopathy, no (\%) & $1(0.7)$ & $1(1.9)$ & $0(0.0)$ & $0(0.0)$ \\
\hline Treatment-related deaths, no $(\%)$ & $0(0.0)$ & $0(0.0)$ & $0(0.0)$ & $0(0.0)$ \\
\hline \multicolumn{5}{|l|}{$\begin{array}{l}\text { Pembrolizumab treatment ongoing } \\
\text { at last follow-up }\end{array}$} \\
\hline Yes, no $(\%)$ & $9(6.5)$ & $4(7.5)$ & $3(3.9)$ & $2(22.2)$ \\
\hline No, no $(\%)$ & $130(93.5)$ & $49(92.5)$ & $74(96.1)$ & $7(77.8)$ \\
\hline \multicolumn{5}{|l|}{ Reason for pembrolizumab discontinuation } \\
\hline Progressive disease, no $(\%)$ & $72(55.4)$ & $23(46.9)$ & $45(60.8)$ & $4(57.1)$ \\
\hline Toxicity, no $(\%)$ & $27(20.8)$ & $9(18.4)$ & $16(21.6)$ & $2(28.6)$ \\
\hline PS decline, no (\%) & $21(16.2)$ & $11(22.4)$ & $10(13.5)$ & $0(0.0)$ \\
\hline Patients own wish, no (\%) & $2(1.5)$ & $1(2.0)$ & $0(0.0)$ & $1(14.3)$ \\
\hline Death, no $(\%)$ & $6(4.6)$ & $5(10.2)$ & $1(1.4)$ & $0(0.0)$ \\
\hline Other, no (\%) & $2(1.5)$ & $0(0.0)$ & $2(2.7)$ & $0(0.0)$ \\
\hline Unknown, no (\%) & $0(0.0)$ & $0(0.0)$ & $0(0.0)$ & $0(0.0)$ \\
\hline
\end{tabular}

Abbreviations: $1 \mathrm{~L}=$ first-line; $2 \mathrm{~L}=$ second-line; $>2 \mathrm{~L}=$ beyond second-line; $\mathrm{CI}=$ confidence interval; $\mathrm{NA}=$ not achieved; $\mathrm{CTCAE}=\mathrm{Common}$ Terminology Criteria for Adverse Events; $\mathrm{AE}=$ adverse event; $\mathrm{PS}=$ performance status. \pm Analysis not performed in this patient group due to low patient number. $\infty$ Number of patients experiencing at least one AE grade 3/4. X Number of patients experiencing a given AE grade $3 / 4$.

was a higher degree of patients with primary bladder tumor than in KEYNOTE-052. In the second-line setting, there was a higher percentage of patients with upper urinary tract tumors than in KEYNOTE-045. This might skew the results towards a shorter survival, due to the poorer prognosis of these patients $[32,33]$.
The relatively low PD-L1 testing rate in the firstline setting could partly be explained by the Danish guidelines in 2018. PD-L1 testing up front in firstline setting was not mandatory before June 2018 at which time preliminary data from Keynote-361 and IMvigor130 indicated reduced survival with immunotherapy, compared with chemotherapy, in 
Table 3

Progression-free survival and overall survival in 139 mUTC patients initiating pembrolizumab at Danish oncology departments in the period March 1, 2018 to February 28, 2019

\begin{tabular}{lcccc}
\hline & All $(n=139)$ & $1 \mathrm{~L}(n=53)$ & $2 \mathrm{~L}(n=77)$ & $>2 \mathrm{~L}(n=9)$ \\
\hline $\begin{array}{l}\text { Time of follow-up, months, } \\
\text { median (range) }\end{array}$ & $20.7(10.7-26.9)$ & 21.1 & & 22.1 \\
PFS, months, median (95\% CI) & $3.5(2.5-5.3)$ & $3.5(2.3-7.9)$ & $2.9(2.5-5.3)$ & - \pm \\
$\begin{array}{l}\text { Death at last follow-up } \\
\quad \text { Yes, no (\%) }\end{array}$ & $95(68.3)$ & $38(71.7)$ & $52(67.5)$ & $5(55.6)$ \\
$\quad$ No, no (\%) & $44(31.7)$ & $15(28.3)$ & $25(32.5)$ & $4(44.4)$ \\
OS, months, median (95\% CI) & $10.0(7.2-13.2)$ & $9.2(7.0-20.9)$ & $9.1(5.4-12.8)$ & $-{ }^{ \pm}$ \\
\hline
\end{tabular}

Abbreviations: $1 \mathrm{~L}=$ first-line; $2 \mathrm{~L}=$ second-line; $>2 \mathrm{~L}=$ beyond second-line; $\mathrm{PFS}=$ progression-free survival; $\mathrm{CI}=$ confidence interval; $\mathrm{OS}=$ overall survival. \pm Analysis not performed in this patient group due to low patient number.

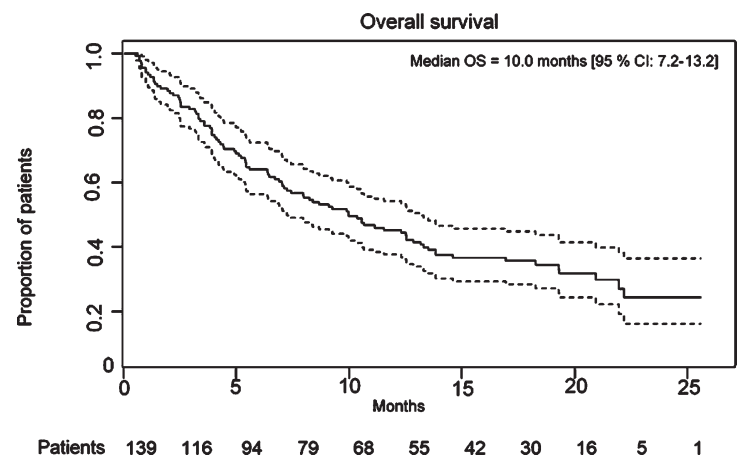

Fig. 1. Overall survival for all treatment lines combined.

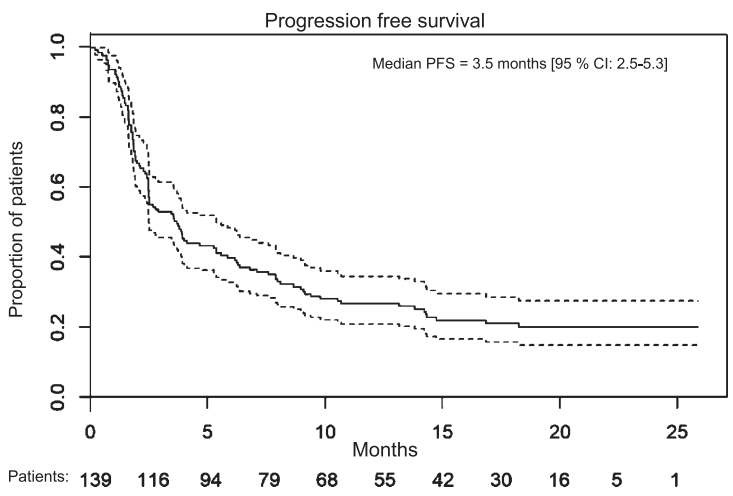

Fig. 2. Progression free survival for all treatment lines combined.

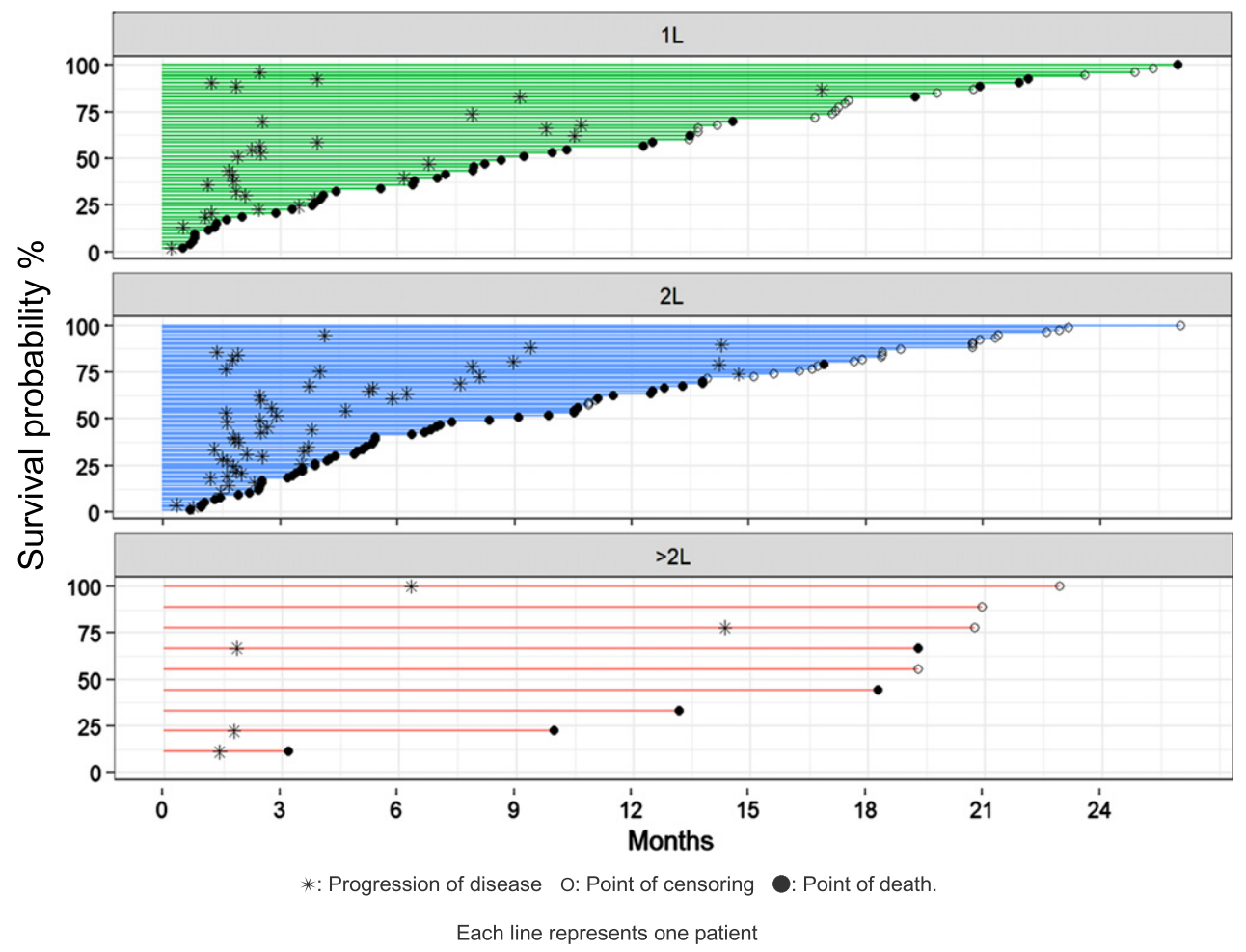

Fig. 3. Swimmer plot showing survival divided by treatment lines. 
Table 4

Comparison of present real-world patient cohort with patient cohorts in the KEYNOTE-052 and KEYNOTE-045 clinical trials

\begin{tabular}{|c|c|c|c|c|}
\hline & $\begin{array}{c}\text { KEYNOTE-052 } \\
(n=370) \\
{[17,20]}\end{array}$ & $\begin{array}{l}\text { RW cohort, } 1 \mathrm{~L} \\
\quad(n=53)\end{array}$ & $\begin{array}{c}\text { KEYNOTE-045 } \\
(n=270) \\
{[13,16]}\end{array}$ & $\begin{array}{l}\text { RW cohort, 2L } \\
\quad(n=77)\end{array}$ \\
\hline $\begin{array}{l}\text { Age at pembrolizumab initiation, } \\
\text { years, median (IQR) }\end{array}$ & 74 & 71 & 67 & 69 \\
\hline \multicolumn{5}{|l|}{ Gender } \\
\hline Male, no (\%) & $286(77)$ & $36(67.9)$ & $200(74.1)$ & $56(72.7)$ \\
\hline Female, no (\%) & $84(23)$ & $17(32.1)$ & $70(25.9)$ & $21(27.3)$ \\
\hline \multicolumn{5}{|l|}{ Smoking status } \\
\hline History of smoking, no (\%) & Not reported & $37(69.8)$ & $165(61.1)$ & $58(75.3)$ \\
\hline No history of smoking, no (\%) & Not reported & $9(17.0)$ & $104(38.5)$ & $9(11.7)$ \\
\hline Unknown, no (\%) & Not reported & $7(13.2)$ & $1(0.4)$ & $10(13.0)$ \\
\hline \multicolumn{5}{|l|}{ Location of primary tumor } \\
\hline Upper urinary tract, no (\%) & $69(19)$ & $5(9.4)$ & $38(14.1)$ & $26(33.8)$ \\
\hline Bladder, no $(\%)$ & $300(81)^{¥}$ & $48(90.6)$ & $232(85.9) ¥$ & $51(66.2)$ \\
\hline Urethra, no $(\%)$ & $0(0)$ & $0(0.0)$ & $0(0.0)$ & $0(0.0)$ \\
\hline Unknown, no (\%) & $1(<1)$ & $0(0.0)$ & $0(0.0)$ & $0(0.0)$ \\
\hline Liver metastases, no (\%) & $78(21)$ & $7(13.2)$ & $91(33.7)$ & $20(26.0)$ \\
\hline \multicolumn{5}{|l|}{ Histology } \\
\hline Urothelial carcinoma, no (\%) & Not reported & $50(94.3)$ & $(100)^{\infty}$ & $73(94.8)$ \\
\hline Squamous cell carcinoma, no (\%) & Not reported & $1(1.9)$ & $0(0.0)$ & $2(2.6)$ \\
\hline Adenocarcinoma, no $(\%)$ & Not reported & $0(0.0)$ & $0(0.0)$ & $0(0.0)$ \\
\hline Mixed histology, no (\%) & Not reported & $2(3.8)$ & $0(0.0)$ & $1(1.3)$ \\
\hline Other, no $(\%)$ & Not reported & $0(0.0)$ & $0(0.0)$ & $1(1.3)$ \\
\hline \multicolumn{5}{|l|}{ ECOG PS at pembrolizumab initiation } \\
\hline 0, no $(\%)$ & $80(22)$ & $14(26.4)$ & $119(44.1)$ & $18(23.4)$ \\
\hline 1, no $(\%)$ & $133(36)$ & $18(34.0)$ & $143(53.0)$ & $45(58.4)$ \\
\hline 2, no $(\%)$ & $156(42)$ & $18(34.0)$ & $2(0.7)$ & $7(9.1)$ \\
\hline 3, no $(\%)$ & $1(<1)$ & $0(0.0)$ & $0(0.0)$ & $0(0.0)$ \\
\hline Unknown, no (\%) & $0(0.0)$ & $3(5.7)$ & $6(2.2)$ & $7(9.1)$ \\
\hline $\begin{array}{l}\text { PD-L1 expression tested prior to } \\
\text { pembrolizumab initiation, no }(\%)\end{array}$ & $357(96)$ & $14(26.4)$ & $260(96.3)$ & $5(6.5)$ \\
\hline \multicolumn{5}{|l|}{ PD-L1 expression (in patients tested) } \\
\hline$<1 \%$, no $(\%)$ & $78(22)$ & $1(7.1)$ & Not reported & $1(20.0)$ \\
\hline $1-9 \%$, no $(\%)$ & $169(47)$ & $0(0.0)$ & Not reported & $0(0.0)$ \\
\hline$\geq 10 \%$, no $(\%)$ & $110(31)$ & $13(92.9)$ & $74(28.5)$ & $4(80.0)$ \\
\hline ORR, no $(\%)$ & $106(28.6)$ & $16(30.2)$ & $57(21.1)$ & $21(27.3)$ \\
\hline Complete response, no (\%) & $33(8.9)$ & $2(3.8)$ & $25(9.3)$ & $4(5.2)$ \\
\hline Partial response, no $(\%)$ & $73(19.7)$ & $14(26.4)$ & $32(11.9)$ & $17(22.1)$ \\
\hline Stable disease, no $(\%)$ & $67(18.1)$ & $7(13.2)$ & $47(17.4)$ & $12(15.6)$ \\
\hline Progressive disease, no $(\%)$ & $157(42.4)$ & $18(34.0)$ & $131(48.5)$ & $32(41.6)$ \\
\hline Mixed response, no $(\%)$ & $0(0)$ & $0(0.0)$ & $0(0.0)$ & $3(3.9)$ \\
\hline No radiologic evaluation performed, no (\%) & $31(8.4)$ & $11(20.8)$ & $35(13.0) €$ & $7(9.1)$ \\
\hline Not evaluable, no $(\%)$ & $9(2.4)$ & $1(1.9)$ & - & $2(2.6)$ \\
\hline Time to response, months, median & 2 & 1.9 & 2.1 & 2.0 \\
\hline Duration of response, months, median & 30.1 & 16.9 & $-\chi$ & 12.9 \\
\hline \multicolumn{5}{|l|}{ PFS } \\
\hline Months, median & 2 & 3.5 & 2.1 & 2.9 \\
\hline 6 months (\%) & 30 & 39.6 & Not reported & 32.5 \\
\hline 12 months (\%) & 22 & 22.6 & 16.8 & 20.8 \\
\hline \multicolumn{5}{|l|}{ OS } \\
\hline Months, median & 11.3 & 9.2 & 10.3 & 9.1 \\
\hline 6 months $(\%)$ & 67 & 66.0 & Not reported & 59.7 \\
\hline 12 months (\%) & 46.9 & 45.3 & 43.9 & 40.0 \\
\hline
\end{tabular}

Abbreviations: $\mathrm{RW}=$ real-world; $1 \mathrm{~L}=$ first-line; $2 \mathrm{~L}=$ second-line; $\mathrm{IQR}=$ interquartile range; ECOG PS = Eastern Cooperative Oncology Group performance status; PD-L1 = programmed death-ligand 1; ORR = overall response rate; $\mathrm{PFS}=$ progression-free survival; OS = overall survival. ${ }^{¥}$ Number of patients with primary tumor in bladder or urethra. ${ }^{\infty}$ Histology showing predominantly transitional-cell features (pure transitional-cell carcinoma $=68.9 \%) . €$ No radiologic evaluation or not evaluable. ${ }^{\chi}$ Median duration of response not reached at time of data cutoff. 
patients with low expressions of PD-L1 [34]. Based on these studies EMA and FDA revised their approval to only recommend immunotherapy to cisplatin-unfit patients with high PD-L1 expression [34, 35]. We included patients from March 1, 2018 through February 28,2019 , and for the first four months all cisplatin ineligible patients regardless of PD-L1 status were eligible for pembrolizumab. However, the rate of PDL1 testing (26.4\%) in the first-line setting, can only partly be explained by this. It is probable that there was a lag-time in implementation of guidelines. In 13 patients, lack of PD-L1 testing can be explained by the patients progressing less than 12 months after neoadjuvant chemotherapy thereby deeming these patients platinum-resistant at the time of diagnosis of advanced disease. Some patients may also have received pembrolizumab without PD-L1 testing, due to physician's choice. Although the number of tested cases is low in this cohort the percentage of cases with PD-L1 > 10\% was much higher in this cohort than in KEYNOTE-052 (92.9\% vs 31\%). This might be due to statistical variability due to the small cohort. More probably the patients tested have been selected for pembrolizumab treatment due to positive PD-L1 test. Two patients have PD-L $1<1 \%$, and still received pembrolizumab, most likely due to treatment before implementation of above-mentioned guidelines.

The ORRs reported in the first-line $(30.2 \%)$ and the second-line (27.3\%) settings of the present study appear a little higher than the ORRs demonstrated in the clinical trials(28.6\% and $21.1 \%$ ) (Table 4) [13, 17]. This is most likely explained by the requirement for central imaging review [13, 17] and for responses being confirmed by follow-up scans after at least four weeks [17] in the clinical trials, in contrast to the real-world setting in which tumor responses are evaluated by CT scans every 12 weeks, without routine review by radiologist peers. Contrarily, in KEYNOTE-52 ORR differs significantly between CPS $<1 \%$ and CPS $>10 \%$ groups [36]. Due to low testing rates in our cohort and hence more diluted CPS, a lower ORR could be expected. Other factors that may explain favorable ORR in our study, might be the lower rate of liver metastases at baseline, slightly lower age and a higher proportion of primary bladder tumors in the first-line setting.

Time to response in the first- and second-line setting was comparable between the present study and the clinical trials. Duration of response for patients enrolled in KEYNOTE-052 (30.1 months median) was almost double that of patients receiving firstline pembrolizumab in the present study (16.9 months median) [20]. The reason for this is not clear, although a possible explanation might be the differences in patient characteristics at baseline, i.e. PD-L1 data is missing for most of our cohort. The treatment guidelines differ between the study populations; patients in the real-world setting are more prone to doctors choice, making patient selection for pembrolizumab more heterogenous than in a trial setting, [11, 29] and RECIST criteria are not systematically used in the real-world setting. Another probable explanation, however, may be the lack of confirmation of progression four weeks after evaluation imaging. This may explain why the lower duration of response, does not translate to comparably lower PFS or OS. Also, pseudo progression, reported with immunotherapy for mUTC between one percent to 17\% [37] can erroneously be interpreted as progression. As median duration of response was not reached in the KEYNOTE-045 trial, comparison with response duration in our patient cohort receiving second-line pembrolizumab is not possible.

Median PFS was 3.5 months (95\% CI: 2.3-7.9 months) for patients receiving first-line pembrolizumab and 2.9 months (95\% CI: 2.5-5.3 months) for patients treated with second-line pembrolizumab. OS was approximately 9 months in the first-line and the second-line treatment setting. In the first-line setting, reported median PFS and the 6 months PFS was higher in our patient cohort than in the KEYNOTE052 trial, which could be due to different tumor assessments being employed in the clinical trial. However, 12 months PFS was comparable between the two groups. In the second-line setting, reported median PFS and PFS at 6 months were slightly higher in our patient cohort than in the KEYNOTE-045 trial. Reported OS in our patient cohort receiving first- and second-line pembrolizumab were marginally lower than OS demonstrated in the clinical trials. This was expected due to the well-known differences between patients treated in clinical trials and the general population [22]. Patients with ECOG PS 2 and one or more of the established poor prognostic factors (i.e., $\mathrm{Hgb}<10 \mathrm{~g} / \mathrm{dl}$, liver metastasis, $<3$ months since last chemotherapy) for second-line therapy were excluded in the KEYNOTE trial but are represented in routine clinical practice and hence our real-world data. Nevertheless, the marginal difference between OS in the KEYNOTE trials and our findings, demonstrates that the results from these clinical trials can be extrapolated to the general mUTC population, which is not always the case in real-world studies [24]. It is noteworthy that most patients responding to treatment 
at 3 months, seem to have durable responses without progression at 6 months, and approximately half have no progression at 12 months, as demonstrated in the swimmers-plot (Fig. 3).

The toxicities registered in our cohort are limited, as data on mild toxicities are not routinely documented. We are therefore only able to report on toxicities CTCAE grade 3-4. We did, however, not find any new toxicities even though the frequency differs from the clinical trials. Approximately 20\% of patients in our cohort registered a grade 3 toxicity, with colitis $(3,6 \%)$ and pneumonitis $(3,6 \%)$ as the most common. Two patients (1.4\%) experienced grade 4 toxicity. The lack of routine registration of toxicities, makes comparison with the clinical trials difficult. In the first-line setting in KEYNOTE-052, $15 \%$ of patients experienced toxicity grade 3,4 or 5 with fatigue being the most common at two percent. Toxicities were broad, and no grade 3, 4 or 5 , toxicities had a frequency above one percent. In KEYNOTE-045, $15 \%$ of patients experienced toxicity of grade 3,4 or 5, hypo- and hyperthyroidism being the most frequent. In our cohort in the secondline setting, $16.9 \%$ of the patients registered a toxicity grade 3 or 4 , and hypo- and hyperthyroidism is not registered as a toxicity in any of these patients. We found a higher frequency of colitis and pneumonitis than in the clinical trials. Our finding matches similar frequencies found with other cancers treated with PD-1 checkpoint inhibitors [38, 39].

The retrospective study design limits the completeness of the collected information. We tried to overcome this and to minimize bias by including treatment centers nationwide with a population-based study design enabling complete inclusion of all mUTC patients treated with pembrolizumab in Denmark. Data on smoking status and ECOG PS are incomplete, as these are not always explicitly noted in the electronic medical records in routine clinical practice. Evaluation of tumor response in the clinical practice is performed by only one radiologist and not with systemic use of RECIST criteria, hence outcomes from the KEYNOTE studies and ours, should be compared with caution. PR and CR are not based on predefined criteria like RESIST in our cohort, and this might be a bias.

Treatment options for mUTC is expanding, and with the many new emerging drugs the last few years, also expected to continuing improving in the future. Even though pembrolizumab is a new addition to the treatment of mUTC, non-responders are frequent, possibly due to the heterogenic nature of the disease, making biological selection markers needed for optimal treatment. Targeted drugs like erdafitinib, [40] enfortumab-vedotin [41] and avelumab maintenance treatment [42] are showing promising results in early trials and real-world data from these novel treatments will be followed with interest. The rapid evolution of targeted therapies offers exciting new options for patients with mUTC.

\section{CONCLUSION}

Real-world data on OS, PFS and toxicity for patients with mUTC receiving pembrolizumab in the first-line and second-line settings, show comparable results to clinical trials. This study further establishes immunotherapy as an effective and tolerable treatment for mUTC.

\section{ACKNOWLEDGMENTS}

The authors have no acknowledgments.

\section{FUNDING}

This research project received funding support from Merck Sharp \& Dohme (MSD) Denmark.

\section{AUTHOR CONTRIBUTIONS}

Omland, Tolver, Pappot and Stormoen had full access to all the data in the study and take responsibility for the integrity of the data and the accuracy of the data analysis. Conception: Pappot, Omland. Acquisition of data: Omland, Carus, Als, Jensen, Pappot. Interpretation of data: Omland, Stormoen, Pappot. Writing the article: Omland, Stormoen, Pappot. Performance of work, Critical revision of the manuscript for important intellectual content: Pappot, Stormoen, Carus, Als, Taarnhøj, Dohn, Jensen. Statistical analysis: Tolver. Obtaining funding: Pappot. Administrative, technical, or material support: Pappot. Supervision: Pappot.

\section{CONFLICT OF INTERESTS}

H.P. has received research grants from Roche, MSD, Pfizer, and Pierre-Fabre. D.R.S has no conflict of interest to report. L.H.O has no conflict of interest to report. A.C. has no conflict of interest to report. G.A.T. has no conflict of interest to report. A.T. has no conflict of interest to report. L.H.D. has no con- 
flict of interest to report. N.V.J. has no conflict of interest to report. A.B.A has no conflict of interest to report.

\section{REFERENCES}

[1] Kamat AM, Hahn NM, Efstathiou JA, Lerner SP, Malmström P-U, Choi W, et al. Bladder cancer. Lancet Lond Engl. 2016;388:2796-810.

[2] von der Maase H, Hansen SW, Roberts JT, Dogliotti L, Oliver T, Moore MJ, et al. Gemcitabine and Cisplatin Versus Methotrexate, Vinblastine, Doxorubicin, and Cisplatin in Advanced or Metastatic Bladder Cancer: Results of a Large, Randomized, Multinational, Multicenter, Phase III Study. J Clin Oncol [Internet]. 2000 [cited 2019 Dec 16];18:3068-77. Available from: http:// ascopubs.org/doi/10.1200/JCO.2000.18.17.3068

[3] Bamias A, Tzannis K, Harshman LC, Crabb SJ, Wong Y-N, Kumar Pal S, et al. Impact of contemporary patterns of chemotherapy utilization on survival in patients with advanced cancer of the urinary tract: a Retrospective International Study of Invasive/Advanced Cancer of the Urothelium (RISC). Ann Oncol [Internet]. 2018 [cited 2019 Dec 16];29:361-9. Available from: https:// academic.oup.com/annonc/article/29/2/361/4565502

[4] De Santis M, Bellmunt J, Mead G, Kerst JM, Leahy M, Maroto P, et al. Randomized Phase II/III Trial Assessing Gemcitabine/Carboplatin and Methotrexate/Carboplatin/Vinblastine in Patients With Advanced Urothelial Cancer Who Are Unfit for Cisplatin-Based Chemotherapy: EORTC Study 30986. J Clin Oncol [Internet]. 2012 [cited 2020 Jan 14];30:191-9. Available from: http://ascopubs.org/doi/10.1200/JCO.2011.37.3571

[5] Omland LH, Lindberg H, Carus A, Als AB, Jensen NV, Taarnh $\varnothing \mathrm{j}$ GA, et al. Real-world Treatment Patterns and Overall Survival in Locally Advanced and Metastatic Urothelial Tract Cancer Patients Treated with Chemotherapy in Denmark in the Preimmunotherapy Era: A Nationwide, Population-based Study. Eur Urol Open Sci. 2021;24:1-8.

[6] Powles T, Durán I, van der Heijden MS, Loriot Y, Vogelzang NJ, De Giorgi U, et al. Atezolizumab versus chemotherapy in patients with platinum-treated locally advanced or metastatic urothelial carcinoma (IMvigor211): a multicentre, open-label, phase 3 randomised controlled trial. The Lancet [Internet]. 2018 [cited 2020 Jul 15];391:748-57. Available from: https://linkinghub.elsevier. com/retrieve/pii/S014067361733297X

[7] McCaffrey JA, Hilton S, Mazumdar M, Sadan S, Kelly WK, Scher HI, et al. Phase II trial of docetaxel in patients with advanced or metastatic transitional-cell carcinoma. J Clin Oncol [Internet]. 1997 [cited 2020 Jul 22];15:1853-7. Available from: http://ascopubs.org/doi/10. 1200/JCO.1997.15.5.1853

[8] Witte RS, Manola J, Burch PA, Kuzel T, Weinshel EL, Loehrer PJ. Topotecan in previously treated advanced urothelial carcinoma: an ECOG phase II trial. Invest New Drugs. 1998;16:191-5.

[9] Lorusso V, Pollera CF, Antimi M, Luporini G, Gridelli C, Frassineti GL, et al. A phase II study of gemcitabine in patients with transitional cell carcinoma of the urinary tract previously treated with platinum. Eur J Cancer [Internet]. 1998 [cited 2020 Jul 22];34:1208-12. Available from:
https://linkinghub.elsevier.com/retrieve/pii/S09598049980 00306

[10] Roth BJ, Manola J, Dreicer R, Graham D, Wilding G, Eastern Cooperative Oncology Group. Piritrexim in advanced, refractory carcinoma of the urothelium (E3896): a phase II trial of the Eastern Cooperative Oncology Group. Invest New Drugs. 2002;20:425-9.

[11] Horwich A, Babjuk M, Bellmunt J, Bruins HM, De Reijke TM, De Santis M, et al. EAU-ESMO consensus statements on the management of advanced and variant bladder cancer-an international collaborative multi-stakeholder effort: under the auspices of the EAU and ESMO Guidelines Committees. Ann Oncol [Internet]. 2019 [cited 2020 Jan 14];30:1697-727. Available from: https:// linkinghub.elsevier.com/retrieve/pii/S0923753420325928

[12] Milowsky MI, Rumble RB, Booth CM, Gilligan T, Eapen LJ, Hauke RJ, et al. Guideline on Muscle-Invasive and Metastatic Bladder Cancer (European Association of Urology Guideline): American Society of Clinical Oncology Clinical Practice Guideline Endorsement. J Clin Oncol Off J Am Soc Clin Oncol. 2016;34:1945-52.

[13] Bellmunt J, de Wit R, Vaughn DJ, Fradet Y, Lee J-L, Fong L, et al. Pembrolizumab as Second-Line Therapy for Advanced Urothelial Carcinoma. N Engl J Med [Internet]. 2017 [cited 2020 Jul 15];376:1015-26. Available from: http://www.nejm.org/doi/10.1056/NEJMoa1613683

[14] Rosenberg JE, Hoffman-Censits J, Powles T, van der Heijden MS, Balar AV, Necchi A, et al. Atezolizumab in patients with locally advanced and metastatic urothelial carcinoma who have progressed following treatment with platinum-based chemotherapy: a single-arm, multicentre, phase 2 trial. The Lancet [Internet]. 2016 [cited 2020 Jul 15];387:1909-20. Available from: https://link inghub.elsevier.com/retrieve/pii/S0140673616005614

[15] Sharma P, Retz M, Siefker-Radtke A, Baron A, Necchi A, Bedke J, et al. Nivolumab in metastatic urothelial carcinoma after platinum therapy (CheckMate 275): a multicentre, single-arm, phase 2 trial. Lancet Oncol [Internet]. 2017 [cited $2020 \mathrm{Jul} 15] ; 18: 312-22$. Available from: https:// linkinghub.elsevier.com/retrieve/pii/S1470204517300657

[16] Fradet Y, Bellmunt J, Vaughn DJ, Lee JL, Fong L, Vogelzang NJ, et al. Randomized phase III KEYNOTE045 trial of pembrolizumab versus paclitaxel, docetaxel, or vinflunine in recurrent advanced urothelial cancer: results of $>2$ years of follow-up. Ann Oncol [Internet]. 2019 [cited 2020 Jul 21];30:970-6. Available from: https:// linkinghub.elsevier.com/retrieve/pii/S0923753419312104

[17] Balar AV, Castellano D, O'Donnell PH, Grivas P, Vuky J, Powles T, et al. First-line pembrolizumab in cisplatinineligible patients with locally advanced and unresectable or metastatic urothelial cancer (KEYNOTE-052): a multicentre, single-arm, phase 2 study. Lancet Oncol [Internet]. 2017 [cited 2020 Mar 27];18:1483-92. Available from: https:// linkinghub.elsevier.com/retrieve/pii/S1470204517306162

[18] Balar AV, Galsky MD, Rosenberg JE, Powles T, Petrylak $\mathrm{DP}$, Bellmunt $\mathrm{J}$, et al. Atezolizumab as first-line treatment in cisplatin-ineligible patients with locally advanced and metastatic urothelial carcinoma: a single-arm, multicentre, phase 2 trial. The Lancet [Internet]. 2017 [cited $2020 \mathrm{Jul}$ 15];389:67-76. Available from: https:// linkinghub.elsevier.com/retrieve/pii/S0140673616324552

[19] https://www.ema.europa.eu/en/news/ema-restricts-usekeytruda-tecentriq-bladder-cancer. EMA;

[20] Vuky J, Balar AV, Castellano D, O’Donnell PH, Grivas P, Bellmunt $\mathrm{J}$, et al. Long-Term Outcomes in KEYNOTE- 
052: Phase II Study Investigating First-Line Pembrolizumab in Cisplatin-Ineligible Patients With Locally Advanced or Metastatic Urothelial Cancer. J Clin Oncol [Internet]. 2020 [cited 2020 Jul 22];JCO.19.01213. Available from: https://ascopubs.org/doi/10.1200/JCO.19.01213

[21] Process and methods guide - how the Danish Medicines Council develops joint regional assessments of the added clinical value of new medicines and new indications Version 1.1 [Internet]. Process Methods Guide - Dan. Med. Counc. Dev. Jt. Reg. Assess. Added Clin. Value New Med. New Indic. Version 11. Available from: https://medicinraadet.dk/ media/re5gfbp5/process-and-methods-guide-join-regionalassessment-of-the-clinical-added-value-of-new-medicinesand-indications-002_adlegacy.pdf

[22] Porzsolt F, Wiedemann F, Becker SI, Rhoads CJ. Inclusion and exclusion criteria and the problem of describing homogeneity of study populations in clinical trials. BMJ Evid-Based Med. 2019;24:92-4.

[23] Nabhan C, Klink A, Prasad V. Real-world Evidence-What Does It Really Mean? JAMA Oncol. 2019;5(6):781-3. doi: 10.1001/jamaoncol.2019.0450.

[24] Sherman RE, Anderson SA, Dal Pan GJ, Gray GW, Gross T, Hunter NL, et al. Real-World Evidence - What Is It and What Can It Tell Us? N Engl J Med [Internet]. 2016 [cited 2020 Jan 13];375:2293-7. Available from: http://www.nejm.org/doi/10.1056/NEJMsb1609216

[25] Saginala K, Barsouk A, Aluru JS, Rawla P, Padala SA, Barsouk A. Epidemiology of Bladder Cancer. Med Sci Basel Switz. 2020;8.

[26] Årsrapport (In Danish). [Internet]. Available from: dablaca.dk

[27] Rosenberg JE, Carroll PR, Small EJ. Update on chemotherapy for advanced bladder cancer. J Urol [Internet]. 2005 [cited 2020 Jun 25];174:14-20. Available from: http://www. jurology.com/doi/10.1097/01.ju.0000162039.38023.5f

[28] Tynkkynen L-K, Alexandersen N, Kaarbøe O, Anell A, Lehto J, Vrangbæk K. Development of voluntary private health insurance in Nordic countries - An exploratory study on country-specific contextual factors. Health Policy Amst Neth. 2018;122:485-92.

[29] Danish Bladder cancer group. Danish national guidelines for treatment of bladder cancer [Internet]. DaBlaCa. 2020. Available from: http://www.skejby.net/DaBlaCaweb/DaBlaCaWEB.htm

[30] Kræftpakkeforløb [Internet]. Available from: https://www. sst.dk/-/media/Udgivelser/2019/Pakkeforloeb-kraeft-20152019/Urinvejene-2019/20190513-Pakkeforloeb-for-kraefti-urinvejene. $a s h x ? \mathrm{la}=\mathrm{da} \& \mathrm{hash}=373505183 \mathrm{AE} 27 \mathrm{~F} 828 \mathrm{CD}$ 742E1D867012D4A170E03

[31] Act on Research Ethics Review of Health Research Projects [Internet]. Available from: https://www.retsinfor mation.dk/eli/lta/2011/593
[32] Sanguedolce F, Cormio L. The complex relationship between upper urinary tract and bladder cancer: clinical and predictive issues. Transl Androl Urol. 2018;7:S248-51.

[33] Green DA, Rink M, Xylinas E, Matin SF, Stenzl A, Roupret $\mathrm{M}$, et al. Urothelial carcinoma of the bladder and the upper tract: disparate twins. J Urol. 2013;189:1214-21.

[34] EMA. EMA restricts use of Keytruda and Tecentriq in bladder cancer [Internet]. Eur. Med. Agency. Available from: https://www.ema.europa.eu/en/news/ema-restrictsuse-keytruda-tecentriq-bladder-cancer

[35] FDA Alerts Health Care Professionals and Oncology Clinical Investigators about an Efficacy Issue Identified in Clinical Trials for Some Patients Taking Keytruda (pembrolizumab) or Tecentriq (atezolizumab) as Monotherapy to Treat Urothelial Cancer with Low Expression of PD-L1 [Internet]. US Food Drug Adm. Available from: https://www.fda.gov/drugs/drug-safety-and-availability/ fda-alerts-health-care-professionals-and-oncologyclinical-investigators-about-efficacy-issue

[36] Balar AV, Castellano D, O'Donnell PH, Grivas P, Vuky J, Powles T, et al. First-line pembrolizumab in cisplatin-ineligible patients with locally advanced and unresectable or metastatic urothelial cancer (KEYNOTE-052): a multicentre, single-arm, phase 2 study. Lancet Oncol. 2017; 18:1483-92.

[37] Kim TJ, Cho KS, Koo KC. Current Status and Future Perspectives of Immunotherapy for Locally Advanced or Metastatic Urothelial Carcinoma: A Comprehensive Review. Cancers. 2020;12:192.

[38] Shohdy KS, Abdel-Rahman O. Risk of pneumonitis with different immune checkpoint inhibitors in NSCLC. Ann Transl Med. 2017;5:365.

[39] Som A, Mandaliya R, Alsaadi D, Farshidpour M, Charabaty A, Malhotra N, et al. Immune checkpoint inhibitor-induced colitis: A comprehensive review. World J Clin Cases. 2019;7:405-18.

[40] Loriot Y, Necchi A, Park SH, Garcia-Donas J, Huddart $\mathrm{R}$, Burgess E, et al. Erdafitinib in Locally Advanced or Metastatic Urothelial Carcinoma. N Engl J Med. 2019;381:338-48.

[41] Rosenberg JE, O'Donnell PH, Balar AV, McGregor BA, Heath EI, Yu EY, et al. Pivotal Trial of Enfortumab Vedotin in Urothelial Carcinoma After Platinum and Anti-Programmed Death 1/Programmed Death Ligand 1 Therapy. J Clin Oncol Off $\mathrm{J}$ Am Soc Clin Oncol. 2019;37:2592-600.

[42] Powles T, Park SH, Voog E, Caserta C, Valderrama BP, Gurney $\mathrm{H}$, et al. Avelumab Maintenance Therapy for Advanced or Metastatic Urothelial Carcinoma. N Engl J Med. 2020;383:1218-30. 